\title{
Quark-meson coupling model with constituent quarks: Exchange and pionic effects
}

\author{
M.E. Bracco ${ }^{a}$, G. Krein ${ }^{b *}$, and M. Nielsen ${ }^{c}$ \\ a Instituto de Física, Universidade do Estado do Rio de Janeiro \\ Rua São Francisco Xavier 524, 20559-900, Rio de Janeiro, RJ - Brazil \\ ${ }^{b}$ Institut für Kernphysik, Universität Mainz, 55099 Mainz, Germany \\ ${ }^{c}$ Instituto de Física, Universidade de São Paulo, Caixa Postal 66318 \\ 05315-970 São Paulo, SP - Brazil
}

\begin{abstract}
The binding energy of nuclear matter including exchange and pionic effects is calculated in a quark-meson coupling model with massive constituent quarks. As in the case with elementary nucleons in QHD, exchange effects are repulsive. However, the coupling of the mesons directly to the quarks in the nucleons introduces a new effect on the exchange energies that provides an extra repulsive contribution to the binding energy. Pionic effects are not small. Implications of such effects on observables are discussed.
\end{abstract}

PACS NUMBERS: 21.65.+f, 24.85.+p, 24.10.Jv, 12.39.-x

KEYWORDS: Quark-meson coupling, nuclear matter, constituent quarks

1. Introduction. In a recent series of papers Saito and Thomas (ST) [1] [2] have developed a model for nuclear matter and finite nuclei in which the quark structure of the nucleons is explicitly considered. The model is based on an original proposal from Guichon [3], and is known as the quark-meson coupling model (QMC). The model shares important features

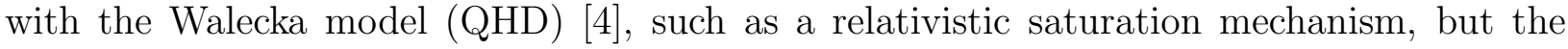
incorporation of explicit quark degrees of freedom has nontrivial consequences (for a list of references see Ref. [2]). Recent developments of the model have been made by Jin and Jennings and Blunden and Miller [0]. For earlier studies within the QMC model see Ref. [6].

\footnotetext{
*Alexander von Humboldt Research Fellow Permanent Address: Instituto de Física Teórica, Universidade Estadual Paulista Rua Pamplona, 145 - 01405-900 São Paulo, SP - Brazil
} 
Exchange and pionic effects have not been investigated so far in the the QMC model. Inclusion of exchange effects (Fock terms) is crucial for assessing the effects of pionic degrees of freedom, which are important in view of the connection of the pion to the dynamical chiral symmetry breaking of QCD. Since the earlier days of QHD, it has been shown [7] that the inclusion of Fock terms requires a sizable renormalization of the parameters of the model, as compared to the values fixed at the mean field level. Different parameters of scalar and vector mesons imply for instance in a different response of matter to external hadrons immersed in medium. Also, the consideration of exchange effects is an initial step towards the consideration of short-range correlations (SRC), which have been shown within the traditional nuclear many-body theory to play an important role on the saturation properties of nuclear matter.

In this paper exchange and pionic effects are studied in the context of a quark-meson coupling model with constituent quarks. The main motivation for using a constituent quark model (CQM) is its simplicity. The CQM has provided a good deal of insight into the study of a variety of hadronic phenomena at the nuclear scale [8], and it is natural to expect that it should be equally useful for addressing the role of quarks on low-energy properties of nuclear matter. In the next section we present the model and discuss approximations. In Section 3 we discuss the Hartree approximation and in Section 4 we consider the Fock terms and study the effects of the pion. Conclusions and future perspectives are presented in Section 5.

2. The model. The mesons are described by the Lagrangian density of QHD, including the $\sigma, \omega, \pi$, and $\rho$ mesons. We separate from the $\sigma, \omega, \rho$ field operators their meanfield, classical values $\phi_{0}, \omega_{0}$ and $\overrightarrow{\rho_{0}}$, and obtain a meson quark-quark interaction from the fluctuating part of the fields. The exchange of mesons between quarks is treated in a similar fashion to the traditional one-gluon interaction [9], with the difference that we do not make a nonrelativistic reduction neither of the kinetic energy nor of the meson-quark interaction. The exchange energy is the expectation value of the meson quark-quark interaction in the state $\mid \Psi>$ of non-overlapping nucleons, which we write in the form of a "Fermi gas of composite nucleons",

$$
\left|\Psi>=\lim _{N \rightarrow \infty} B_{\alpha_{1}}^{\dagger}\left(\boldsymbol{p}_{1}\right) \cdots B_{\alpha_{N}}^{\dagger}\left(\boldsymbol{p}_{N}\right)\right| 0>
$$

where the nucleon creation operator is given by

$$
B_{\alpha}^{\dagger}(\boldsymbol{p})=\frac{1}{\sqrt{3 !}} \frac{\epsilon_{i j k}}{\sqrt{3 !}} \frac{T_{\alpha}^{a b c}}{\sqrt{18}} \int d \boldsymbol{k}_{1} d \boldsymbol{k}_{2} \boldsymbol{k}_{3} \Phi_{\boldsymbol{p}}\left(\boldsymbol{k}_{1}, \boldsymbol{k}_{2}, \boldsymbol{k}_{3}\right) q_{a}^{i \dagger}\left(\boldsymbol{k}_{1}\right) q_{b}^{j \dagger}\left(\boldsymbol{k}_{2}\right) q_{c}^{k \dagger}\left(\boldsymbol{k}_{3}\right)
$$


$\Phi_{\boldsymbol{p}}$ is the three-quark Fock-space amplitude, where $\boldsymbol{p}$ is the nucleon center of mass (c.m.) momentum, and $\alpha$ the nucleon spin-isospin third components. $T_{\alpha}^{a b c}$ is a spin-isospin ClebschGordon coefficient, $a, b, c=(s f)$ are the quark spin-flavor quantum numbers and $\epsilon_{i j k}$ is the totally antisymmetric tensor in the color indices. The quark creation and annihilation operators satisfy canonical anticommutation relations. The largest value of $|\boldsymbol{p}|$ in Eq (1) is the nucleon Fermi momentum $k_{F}$, which is related to the nuclear matter density as $\rho_{N}=$ $\gamma k_{F}^{3} / 6 \pi^{2}$, with $\gamma=4(2)$ for nuclear (neutron) matter.

Let us briefly consider the nucleon in free space, and collect some useful material for later discussions. For simplicity, we neglect gluons and use for the confining potential an harmonic oscillator [8], $V(r)=-\lambda^{a} \lambda^{a} / 4 C r^{2} / 2$. With a nonrelativistic kinetic energy, the problem is exactly soluble. For the ground state,

$$
\Phi_{\boldsymbol{p}}\left(\boldsymbol{k}_{1}, \boldsymbol{k}_{2}, \boldsymbol{k}_{3}\right)=\delta\left(\boldsymbol{p}-\boldsymbol{k}_{1}-\boldsymbol{k}_{2}-\boldsymbol{k}_{3}\right)\left(\frac{3 b^{4}}{\pi^{2}}\right)^{3 / 4} e^{-b^{2} / 6 \sum_{i<j}^{3}\left(\boldsymbol{k}_{i}-\boldsymbol{k}_{j}\right)^{2}}
$$

where $b$ is the r.m.s. radius of the nucleon. In case of a relativistic kinetic energy, the problem is not exactly soluble. We then use $\Phi$ given as above, and treat $b$ as a variational parameter. In this case, the ground state mass of the nucleon is given by

$$
M_{N}=\left(\frac{3 b^{2}}{2 \pi}\right)^{3 / 2} \int d \boldsymbol{k} \sqrt{\boldsymbol{k}^{2}+m_{q}^{2}}\left[3+\frac{9}{2}\left(b^{2} \boldsymbol{k}^{2}-1\right)\right] e^{-3 b^{2} \boldsymbol{k}^{2} / 2},
$$

where the second term in the square bracket is the energy from the confining potential, $3 C b^{2}$. The constant $C$ was eliminated making use of the variational condition on $M_{N}$.

In order to calculate the energy density of nuclear matter $\mathcal{E}$ we need the interaction of the quarks with the unconfined, mean meson fields. We obtain the meson quark-quark interaction in a similar fashion to the derivation of the one-gluon interaction in the CQM [9], but with the difference that we use Dirac field operators expanded in a plane-wave basis, $\psi_{f}^{i}(x)=\int d \boldsymbol{k} /(2 \pi)^{3 / 2} \sum_{s} u_{s}(\boldsymbol{k}) e^{i k \cdot x} q_{s f}^{i}(\boldsymbol{k})$, with

$$
u_{s}(\boldsymbol{k})=\sqrt{\frac{E^{*}(\boldsymbol{k})+m_{q}^{*}}{2 E^{*}(\boldsymbol{k})}}\left(\begin{array}{c}
1 \\
\frac{\boldsymbol{\sigma} \cdot \boldsymbol{k}}{E^{*}(\boldsymbol{k})+m_{q}^{*}}
\end{array}\right) \chi_{s}
$$

where $E^{*}(\boldsymbol{k})=\sqrt{\boldsymbol{k}^{2}+m_{q}^{* 2}}$, and $m_{q}^{*} \equiv m_{q}-g_{q \sigma} \phi_{0}$ is determined requiring stability of the energy density with respect to variations in $\phi_{0}$. Note that since antiquarks are neglected, the three-quark nucleon wavefunction, $<\psi\left(x_{1}\right) \psi\left(x_{2}\right) \psi\left(x_{3}\right) \mid B_{\alpha}^{\dagger}(\boldsymbol{p})>$, contains only positiveenergy components. This ensures that the Dirac Hamiltonian is bounded from below. We 
note however that neglecting antiquarks is not entirely consistent since it does not lead to a well defined Hilbert space, and should be considered as part of the definition of the model. The use of a wave function with a complete Dirac structure requires the use of a different variational principle to avoid the continuum dissolution problem [10], such as the minimax variational principles used in atomic physics for relativistic many-electron systems [11]. In this case, the energy functional in the space of variational parameters is a minimum with respect to the parameters related to the upper component of the Dirac spinor, and a maximum with respect to the ones related to the lower component. Such variational calculations have been recently applied to quarks models [12], and appear to be particularly suitable for nuclear matter calculations like the present one, where a variational calculation seems to be imperative. We do not pursue this here, rather reserve it for future elaboration, along with the consideration of a proper relativistic structure for the confining potential. This last point is still a matter actively studied [13]; it is relevant in connection with spin- and momentum-dependent effects of the confining interaction, which are not considered in the present paper. In the approximation we are using, there is no difference between a potential that is the time component of a four-vector and a scalar potential in which all spin and momentum-dependent terms are neglected in a nonrelativistic expansion. For a wave function with a complete Dirac structure however, a confining vector interaction would lead to problems with the Klein paradox, as is well known [14]. One should also note that when Fock terms are considered, Eq. (5) is not the most general spinor basis, since exchange effects induce a momentum dependence for the quark self-energies. In this sense, our approach shares similarities with the one of Chin [7] in QHD, where exchange energies are evaluated with the mean field self-energies. The same approximation is employed in the Dirac-Brueckner approach [15].

For nucleons in medium, we use a gaussian form for $\Phi_{\boldsymbol{p}}$ as in Eq. (3), with $b$ replaced by a $b^{*}$, and determine $b^{*}$ variationally. Neglecting effects due to the superposition of the quark clusters in medium, such as quark exchanges between different nucleons (these are discussed in Section 4) and effects thereof [16], the energy density of nuclear matter is given by

$$
\begin{aligned}
\mathcal{E} & =\gamma\left(\frac{3 b^{* 2}}{2 \pi}\right)^{3 / 2} \int_{0}^{k_{F}} \frac{d \boldsymbol{p}}{(2 \pi)^{3}} \int d \boldsymbol{k}\left[3 \sqrt{\boldsymbol{k}^{2}+m_{q}^{* 2}}+3 C b^{* 2}\right] e^{-3 b^{* 2}(\boldsymbol{k}-\boldsymbol{p} / 3)^{2} / 2} \\
& +\frac{1}{2} m_{\sigma}^{2} \phi_{0}^{2}+\frac{1}{2} m_{\omega}^{2} \omega_{0}^{2}+\frac{1}{2} m_{\rho}^{2} \vec{\rho}_{0}^{2}+\sum_{p=\sigma \omega \pi \rho} \mathcal{V}_{F}^{p}
\end{aligned}
$$

where $\mathcal{V}_{F}^{p}$ is the mean field exchange energy 


$$
\mathcal{V}_{F}^{p}=+\frac{9}{2} \int_{0}^{k_{F}} \frac{d \boldsymbol{p}}{(2 \pi)^{3}} \int_{0}^{k_{F}} \frac{d \boldsymbol{p}^{\prime}}{(2 \pi)^{3}} \omega_{p}\left(\boldsymbol{p}, \boldsymbol{p}^{\prime}\right) e^{-b^{* 2}\left(\boldsymbol{p}^{\prime}-\boldsymbol{p}\right)^{2} / 3}
$$

where

$$
\begin{aligned}
& w_{p}\left(\boldsymbol{p}, \boldsymbol{p}^{\prime}\right)=\left(\frac{3 b^{* 2}}{2 \pi}\right)^{3} \int d \boldsymbol{k} d \boldsymbol{q} e^{-3 b^{* 2} / 2\left[(\boldsymbol{k}-\overline{\boldsymbol{k}})^{2}+(\boldsymbol{q}-\overline{\boldsymbol{q}})^{2}\right]} \Delta_{p}\left(\boldsymbol{t} ; \delta E^{*}(\boldsymbol{k}, \boldsymbol{t})\right) \frac{T_{\alpha}^{\left(s_{4} f_{4}\right) b_{2} c_{2}}}{\sqrt{18}} \\
& \times\left[\bar{u}_{s_{4}}(\boldsymbol{k})\left(\Gamma^{p}\right)^{f_{4} f_{2}} u_{s_{2}}(\boldsymbol{k}+\boldsymbol{t})\right] \frac{T_{\beta}^{\left(s_{2} f_{2}\right) b_{2} c_{2}}}{\sqrt{18}} \frac{T_{\beta}^{\left(s_{3} f_{3}\right) b_{1} c_{1}}}{\sqrt{18}}\left[\bar{u}_{s_{3}}(\boldsymbol{q})\left(\Gamma_{p}\right)^{f_{3} f_{1}} u_{s_{1}}(\boldsymbol{q}-\boldsymbol{t})\right] \frac{T_{\alpha}^{\left(s_{1} f_{1}\right) b_{1} c_{1}}}{\sqrt{18}},
\end{aligned}
$$

where $\boldsymbol{t}=\boldsymbol{p}^{\prime}-\boldsymbol{p}, \overline{\boldsymbol{k}}=(\boldsymbol{p}-\boldsymbol{t}) / 3, \overline{\boldsymbol{q}}=\left(\boldsymbol{p}^{\prime}+\boldsymbol{t}\right) / 3, \delta E^{*}(\boldsymbol{k}, \boldsymbol{t})=E^{*}(\boldsymbol{k})-E^{*}(\boldsymbol{k}+\boldsymbol{t})$, and the vertices and propagators are

$$
\begin{aligned}
& \Gamma^{\sigma}=i g_{q \sigma}, \quad \Gamma^{\omega}=-i g_{q \omega} \gamma^{\mu}, \quad \Gamma_{p v}^{\pi}=\frac{f_{q \pi}}{m_{\pi}} \not q \gamma^{5} \tau^{a}, \quad \Gamma^{\rho}=\left(-i g_{q \rho} \gamma^{\mu}-\frac{f_{q \rho}}{2 m_{q}} \sigma^{\mu \nu} q_{\nu}\right) \tau^{a}, \\
& \Delta_{\sigma, \pi}(\boldsymbol{k} ; E(\boldsymbol{k}))=\frac{1}{E^{2}(\boldsymbol{k})-\boldsymbol{k}^{2}-m_{\sigma, \pi}^{2}}, \quad \Delta_{\omega, \rho}(\boldsymbol{k}, E(\boldsymbol{k}))=\frac{-1}{E^{2}(\boldsymbol{k})-\boldsymbol{k}^{2}-m_{\omega, \rho}^{2}},
\end{aligned}
$$

where $q=k_{3}-k_{1}=k_{2}-k_{4}$. We have dropped the term proportional to $k^{\mu} k^{\nu} / m_{\omega, \rho}^{2}$ in the vector potential because of current conservation.

As the final step, $m_{q}^{*}$ and $b^{*}$ are determined through the variational equations $\partial \mathcal{E} / \partial \phi_{0}=0$ and $\partial \mathcal{E} / \partial b^{*}=0$. Note that $\phi_{0}$ is a dynamical variable, and as such can always be determined from the thermodynamic argument that an isolated system at fixed baryon number and volume (and zero temperature) will minimize its energy.

3. Hartree Approximation. The Hartree approximation consists in taking $\mathcal{V}_{F}=0$ in Eq. (6). The minimization of the energy density with respect to $\phi_{0}$ leads to

$$
3 m_{q}^{*}=3 m_{q}-2 \frac{9 g_{q \sigma}^{2}}{2 m_{\sigma}^{2}} \gamma\left(\frac{3 b^{* 2}}{2 \pi}\right)^{3 / 2} \int_{0}^{k_{F}} \frac{d \boldsymbol{p}}{(2 \pi)^{3}} \int d \boldsymbol{k} \frac{m_{q}^{*}}{\sqrt{\boldsymbol{k}^{2}+m_{q}^{* 2}}} e^{-3 b^{* 2}(\boldsymbol{k}-\boldsymbol{p} / 3)^{2} / 2}
$$

Before discussing the numerical results, we start performing a nonrelativistic approximation, with the only purpose of getting insight into the problem. All integrals can be done analytically. Eq. (11) becomes

$$
3 m_{q}^{*}=3 m_{q}-2 \frac{9 g_{q \sigma}^{2}}{2 m_{\sigma}^{2}}\left\{\left[1-\frac{3}{5} \frac{k_{F}^{2}}{2\left(3 m_{q}^{*}\right)^{2}}\right]-\frac{1}{2 m_{q}^{* 2} b^{* 2}}\right\} \rho_{N},
$$

and the energy per nucleon (for symmetrical nuclear matter $\vec{\rho}_{0}=0$ )

$$
\begin{aligned}
\frac{E}{A}-M_{N} & =3\left(m_{q}^{*}-m_{q}\right)+\frac{3}{2 m_{q} b^{2}}\left(\frac{b^{* 2}}{b^{2}}+\frac{m_{q}}{m_{q}^{*}} \frac{b^{2}}{b^{* 2}}-2\right)+\frac{3}{5} \frac{k_{F}^{2}}{2\left(3 m_{q}^{*}\right)} \\
& +\frac{1}{4} \frac{2 m_{\sigma}^{2}}{9 g_{q \sigma}^{2}}\left(3 m_{q}-3 m_{q}^{*}\right)^{2} \frac{1}{\rho_{N}}+\frac{9 g_{q \omega}^{2}}{2 m_{q \omega}^{2}} \rho_{N} .
\end{aligned}
$$


The variational condition for $b^{*}$ leads to $b^{*} / b=\left(m_{q} / m_{q}^{*}\right)^{1 / 4}>1$, since $m_{q}^{*} / m_{q}<1$. An interesting comparison can be made with QHD, when the Dirac spinors for the nucleons are expanded to $\mathcal{O}\left(\boldsymbol{p}^{2} / M_{N}^{2}\right)$ [17]. The effective mass of the nucleon, Eq. (11) of Ref. [17], becomes $M_{N}^{*}=M_{N}-g_{\sigma}^{2} / m_{\sigma}^{2}\left(1-3 k_{F}^{2} / 10 M_{N}^{* 2}\right) \rho_{N}$. Comparing this with Eq. (12) above, it becomes evident that the effect of internal structure of the nucleon is the term $1 / 2 m_{q}^{* 2} b^{* 2}$ in Eq. (12). This term comes from the kinetic energy of the quarks in the nucleon, and is of the opposite sign to the QHD term. The internal motion of the quarks provides an extra repulsion and acts as a repulsive field in medium.

We next consider the numerical solutions of the relativistic equations. In this case the integrals in Eqs. (6) (77)(8) and (11) must be performed numerically. The determination of $b^{*}$ must also be done numerically. We fix the coupling constants such as to obtain a stable minimum of $E / N-M_{N} \simeq-15.75 \mathrm{MeV}$ at $k_{F} \simeq 1.36 \mathrm{fm}^{-1}$. We use standard values for the masses, namely $m_{q}=350 \mathrm{MeV}, m_{\sigma}=550 \mathrm{MeV}$, and $m_{\omega}=783 \mathrm{MeV}$. The parameter set is shown in Table I, where we show results for $b=0.6 \mathrm{fm}$ and $b=0.7 \mathrm{fm}$.

In Fig. 1 we plot $E / N-M_{N}$ as a function of $k_{F}$ for different model parameters of Table I. In Fig. 1(a), the QHD mean-field result with parameter set (1) in Table I, is the long-dashed line. Our Hartree result for $b=0.6 \mathrm{fm}$, parameter set (2), is the long-short-dashed line. Inspection of Fig. 1(a) reveals that our Hartree result provides a nuclear compressibility, $K$, that is smaller than the one obtained in QHD. As seen in Table I, the values for $K$ for $b=0.6 \mathrm{fm}$ and $b=0.7 \mathrm{fm}$ are considerable smaller than the value in QHD (MFT), $K=545 \mathrm{MeV}$. The qualitative feature of the results is that the effective repulsion provided by the internal degrees of freedom of the nucleons increases as $b$ decreases.

The original QMC model of ST predicted a much smaller mean $\omega$ field as predicted in finite-density QCD sum rules and relativistic nuclear phenomenology [18. On the other hand, when the correction due to the c.m. in the ST model is taken to be independent of the applied field, the discrepancies are significantly reduced. Our value of $g_{\omega}$ is very similar to the new value in the ST model [2].

3. Exchange and pionic contributions. The evaluation of the Fock energy involves the summation over the spin-isospin quantum numbers of the quarks and the evaluation of a seven-dimensional numerical integration in Eqs. (7) (8). The spin-isospin sum can be easily performed using the "substitution rules" [19], which allow to express quark spin-isospin Pauli matrices in terms of nucleon spin-isospin Pauli matrices. Regarding the multidimensional integral, although it can be carried out using a Monte Carlo integrator, we notice that the 
integrands of the $\boldsymbol{k}$ and $\boldsymbol{q}$ integrals in Eq. (8) are concentrated around the values of $\overline{\boldsymbol{k}}$ and $\overline{\boldsymbol{q}}$, respectively . In view of this, we use $\overline{\boldsymbol{k}}$ for $\boldsymbol{k}$ and $\overline{\boldsymbol{q}}$ for $\boldsymbol{q}$ in the spinors and in the meson propagators and integrate analytically over $\boldsymbol{q}$ and $\boldsymbol{k}$. What remains is a three-dimensional integral, which has to be performed numerically. The minimization of the energy density with respect to $\phi_{0}$ and $b^{*}$ is also performed numerically.

The new values of $g_{\sigma}$ and $g_{\omega}$ are shown in Table I. The readjustment of $g_{\omega}$ is relatively small, of the order of $-10 \%$, and of $g_{\sigma}$ is of the order of $+40 \%$. As in QHD, the effect of the Fock terms is repulsive, as seen in Fig. 1(a), where the curve with short-dashes is calculated with the Hartree parameter set (2), including the exchange terms from the $\sigma$ and $\omega$ mesons. However, the internal structure of the nucleon has an extra, nontrivial effect, which can be qualitatively understood through a nonrelativistic reduction of the Fock energy from the $\sigma$ meson

$$
\mathcal{V}_{F}^{s}=+\frac{1}{2} 9 g_{q \sigma}^{2} \gamma \int_{0}^{k_{F}} \frac{d \boldsymbol{p}}{(2 \pi)^{3}} \int_{0}^{k_{F}} \frac{d \boldsymbol{p}^{\prime}}{(2 \pi)^{3}} \frac{e^{-b^{* 2}\left(\boldsymbol{p}-\boldsymbol{p}^{\prime}\right) / 3}}{\left(\boldsymbol{p}-\boldsymbol{p}^{\prime}\right)^{2}+m_{s}^{2}}\left\{\left[1-\frac{\left(\boldsymbol{p}+\boldsymbol{p}^{\prime}\right)^{2}}{4\left(3 m_{q}^{*}\right)^{2}}\right]-\frac{1}{m_{q}^{* 2} b^{2}}\right\} .
$$

The first term in square brackets is the nonrelativistic reduction of the exchange energy in QHD [7]. The term $1 / m_{q}^{* 2} b^{2}$ represents the nontrivial effect of the internal structure of the nucleon, which is of the opposite sign to the QHD term, and is responsible for the enhancement of $g_{\sigma}$. This term is equivalent to the $\sigma$ dependence of the $g_{\sigma N}$ coupling constant in the original QMC work [1]. A similar, but smaller effect is seen in the contribution from the $\omega$. The enhancement of $g_{\sigma}$ is of interest for a good description of the spin-orbit splittings in finite nuclei [2].

The $\pi$-quark coupling constant is determined [19] by comparing the asymptotic behavior of the nucleon-nucleon one-pion-exchange potentials at the quark level and for point nucleons: $f_{q \pi}^{2}=(9 / 25) f_{N \pi}^{2} \exp \left(-m_{\pi}^{2} b^{2} / 2\right)$, with $f_{N \pi}^{2}=g_{N \pi}^{2}\left(m_{\pi}^{2} / 4 M_{N}^{2}\right)=0.98, m_{\pi}=138 \mathrm{MeV}$ and $g_{N \pi}^{2} / 4 \pi=14.4$. The effect of the $\pi$ is not small, although somewhat smaller than the exchange effects from the $\sigma$ and $\omega$ mesons. The dash-dotted curve is calculated with the $\mathrm{H}$ parameter set (2), and includes the exchange effects of the $\sigma$ and $\omega$. The new readjustment of $g_{\sigma}$ and $g_{\omega}$ to saturate nuclear matter at the right place is relatively small, as seen in Table I, entry (5).

Because of the large tensor coupling of the $\rho$ meson, which is of the comparable magnitude to the $\pi$ coupling, we also include its effects in the present calculation. The $\rho$-quark couplings are fixed as in the case of the $\pi$. For comparison with the results of Ref. [20], we use $g_{N \rho}^{2} / 4 \pi=0.55, f_{N \rho} / g_{N \rho}=3.7$, and $m_{\rho}=770 \mathrm{MeV}$. The effect of the $\rho$ is also repulsive. 
The new values for $g_{\sigma}$ and $g_{\omega}$ are given by the parameter set (6) in Table I. The solid curve in Fig. 1(a) is the result of including all mesons.

As in QHD [4], the effect of the $\pi$ and the tensor coupling of the $\rho$ are repulsive. This is due to the zero-range (ZR) components in the interaction, which would not contribute if short-range correlations are taken into account. In order to obtain a more reasonable estimate of the effects of $\pi$ and $\rho$, we follow Ref. [20] and remove this component from the interaction. The effect provided by the attractive $\pi$ is of the order of $10 \mathrm{MeV}$, similar do the effect in the QHD calculation of Ref. [20]. The long-dashed curve in Fig. 1(b) is calculated with the $\mathrm{H}$ parameter set (2) and the ZR component of the $\pi$ removed, and includes the exchange terms of $\sigma$ and $\omega$ mesons. The long-short-dashed curve in Fig. 1(b) is calculated with the H parameter set (2) and the ZR components from both the $\pi$ and the $\rho$ interactions removed. The net attraction provided by the $\pi$ and $\rho$ is of the order of $24 \mathrm{MeV}$ per nucleon.In the calculation of Ref. [20], for the same value of $f_{N \rho} / g_{N \rho}=3.7$, the net attraction is of the order of $26 \mathrm{MeV}$ per nucleon.

To conclude, we note that the exchange effects considered here arise from the Pauli principle at the nucleon level, i.e. quark-exchange (QE) between different nucleons were neglected. QE effects are expected to be small at low densities, but for for nucleons with $b=0.6 \mathrm{fm}$ in a medium at the normal nuclear density, they might have interesting consequences. In particular, they might be of interest for replacing $\omega$ exchange as the main source of the short-range repulsion, in view of possible conceptual difficulties with the coupling of an extended meson to quarks inside the nucleons [1] [3]. There is an extensive literature [21] in this subject and it is out of the scope of the present paper to review the subject here. The present model can be naturally extended to include QE effects following the idea [19] that the innermost, short-ranged part of the NN force is generated by QE with pion coupling, and the outer part is generated by meson coupling to the nucleon. In such a picture, there is no danger for double counting by taking simultaneously QE and $\omega$ exchange, since the $\omega$ coupling to the nucleon is cut-off by the nucleon form factor. We use the approach of Ref. 22 to derive an effective NN interaction from pion-quark exchange. The resulting NN interaction is non-local and gives scattering phase-shifts numerically similar to the ones obtained with the resonating group method [21]. After a long calculation, with the extensive use of the substitution rules of Ref. [19], the contribution of QE to the energy density of symmetrical nuclear matter can be written as

$$
\mathcal{V}_{q}^{e x c h}=\frac{f_{q \pi}^{2}}{3 m_{\pi}^{2}} \int_{0}^{k_{F}} \frac{d \boldsymbol{p}}{(2 \pi)^{3}} \int_{0}^{k_{F}} \frac{d \boldsymbol{p}^{\prime}}{(2 \pi)^{3}} \sum_{k=1}^{5} A_{k} e^{-a_{k} b^{* 2}\left(\boldsymbol{p}-\boldsymbol{p}^{\prime}\right)^{2}}
$$


with $A_{1}=8(3 / 4)^{3 / 2}, a_{1}=1 / 12, A_{2}=54, a_{2}=0, A_{3}=120(12 / 11)^{3 / 2}, a_{3}=2 / 33$, $A_{4}=-44 / 3, a_{4}=1 / 3, A_{5}=-272 / 3(12 / 11)^{3 / 2}, a_{5}=8 / 33$. Here, only the contribution of the zero-range part of the pion-exchange is shown. The contribution from the long-range part is numerically negligible, as also found in Ref. [19], where a nonrelativistic form for the pion-exchange was used. The new coupling constants (we do not consider $\rho$ coupling) are $g_{\sigma}^{2}=173.45$ and $g_{\omega}^{2}=56.2$. The compressibility is $K=375 \mathrm{MeV}$ and $b^{*} / b=1.07$. Note that this value of $g_{\omega}^{2}$ is very close to the quark-model SU(6) symmetry prediction $g_{\omega}^{2} / 4 \pi \approx 9 g_{\rho}^{2} / 4 \pi=9 \times 0.55$, which is much smaller than the values used in QHD and in one-boson-exchange models [15]. We have also checked the effect of quark exchange with $\sigma$ coupling; the effect amounts to a new readjustment of $g_{\sigma}$, with no important consequences for the values of $K$ and $b^{*} / b$.

4. Conclusions and Future Perspectives. The consideration of exchange effects in QMC-type of models is the initial step towards the implementation of chiral symmetry and short-range correlations. Important consequences are expected when both elements are put together, as recently shown by Banerjee and Tjon [23] in a study using density-dependent meson masses and couplings. We have shown here that the coupling of mesons directly to the quarks has important consequences on the exchange energy, implying in a sizable readjustment of the meson-quark coupling constants. A readjustment of coupling constants is not meaningless, since different coupling constants imply in different in-medium spin-spin, spin-orbit and tensor forces and therefore different responses will be experienced by external hadrons immersed in medium.

An interesting new direction is the replacement of $\omega$-coupling by nucleon overlap at short distances. The idea of describing the very short-range part of the NN interaction by quark-exchange has long been discussed and we have shown that an interesting consequence is a large reduction of the value of $g_{\omega}$. Another interesting aspect that is expected to influence the saturation mechanism is the inclusion of the low-lying nucleon resonances. The present model has the potentiality to incorporate such effects, since with the values $\hbar \omega=1 / m_{q}^{2} b^{2}=174 \mathrm{MeV}$ and $g_{q \pi}$ used here it is possible to obtain a reasonably good description of the low-mass spectrum of the nucleon, including the Roper resonance [24].

An important and necessary next step is to improve on the Lorentz transformation properties of QMC-type of models, based on bag or semi-relativistic constituent quark models, a problem that is intimately related to the projection of the c.m. motion. This is because low 
energy theorems [25] based on Lorentz invariance impose constraints on the single-nucleon energy in matter which have a direct impact on the saturation properties of nuclear matter.

Acknowledgments. GK thanks R. Brockmann for discussions and suggestions. GK also thanks G.A. Miller and A.W. Thomas for discussions on the QMC model during the program "Quark and gluon structure of nucleons and nuclei" of the National Institute for Nuclear Theory in Seattle. This work was supported in part by the Alexander von Humboldt Foundation (Germany) and FAPESP and CNPq (Brazil). 


\section{REFERENCES}

[1] K. Saito and A. W. Thomas, Phys. Lett. B327 (1994) 9.

[2] K. Saito, K. Tsushima and A. W. Thomas, Nucl.Phys. A609 (1996) 339.

[3] P.A.M. Guichon, Phys. Lett. B200 (1988) 235.

[4] B.D. Serot and J. D. Walecka, Adv. Nucl. Phys. 16 (1986) 1.

[5] X. Jin and B.K. Jennings, Phys. Lett. B374 (1996) 13; Phys. Rev. C54 (1996) 1427;

P. Blunden and G.A. Miller, Phys. Rev. C54 (1996) 359.

[6] T. Frederico, B.V. Carlson, R.A. Rego and M.S. Hussein, J. Phys. G15 (1989) 397;

S. Fleck, W. Bentz, K. Shimizu and K. Yazaki, Nucl. Phys. A510 (1990) 731; E. Naar and M.C. Birse, J. Phys. G 19 (1993) 555.

[7] S.A. Chin, Ann. Phys. 108 (1977) 301.

[8] A. Le Yaouanc, LL. Oliver, O. Pène and J.-C. Raynal, Hadron Transitions in the Quark Model, Gordon and Breach Science Publishers, Amsterdam (1988).

[9] A. de Rujula, H. Georgi and S. Glashow, Phys. Rev. D12 (1975) 147; S. Capstick and N. Isgur, Phys. Rev D34 (1990) 2809.

[10] G.E. Brown and D.G. Ravenhall, Proc. Roy. Soc. London, Ser. A 208 (1951) 552.

[11] J.D. Talman, Phys. Rev. Lett. 57 (1986) 1091.

[12] J. Giammarco and J. Franklin, Nucl. Phys. A585 (1995) 450.

[13] A.P. Szczepaniak and E.S. Swanson, Phys. Rev. D55 (1997) 3987.

[14] P. Fishbane, S. Gasiorowicz, D. Johannsen and P. Kaus, Phys. Rev. D27 (1983) 2433.

[15] R. Brockmann and R. Machleidt, Phys. Rev. C 42 (1990) 1965.

[16] G. Krein and Th.A.J. Maris, Phys. Rev. C36 (1987) 365.

[17] P. Amore, M.B. Barbaro and A. de Pace, Phys. Rev. C53 (1996) 2801.

[18] T. D. Cohen, R. J. Furnstahl, D. K. Griegel and X. Jin, Prog. Part. Nucl. Phys. 35 (1995) 221; X. Jin, M. Nielsen, T. D. Cohen, R. J. Furnstahl and D. K. Griegel, Phys. Rev. C49 (1994) 464; S. J. Wallace, Ann. Rev. Nucl. Part. Sci. 37 (1987) 267.

[19] G.Q. Liu, M. Swift, A. W. Thomas and K. Holinde, Nucl. Phys. A556 (1993) 331.

[20] A. Bouyssy, J.-F. Mathiot, N. Van Giai and S. Marcos, Phys. Rev. C36 (1987) 380.

[21] K. Yazaki, Prog. Part. Nucl. Phys. 24 (1990) 353.

[22] D. Hadjimichef, G. Krein, S. Szpigel and J.S. da Veiga, Phys. Lett. B367 (1996) 317.

[23] M. Banerjee and J. A. Tjon, Phys. Rev. C 56 (1997) 497.

[24] L.Ya. Glozman and D.O. Riska, Phys. Rep. 268 (1996) 263.

[25] S.J. Wallace, F. Gross and J.A. Tjon, Phys. Rev. Lett. 74 (1995) 228; D.R. Phillips, M.C. Birse and S.J. Wallace, Phys. Rev. C55 (1997) 1937. 
TABLE I: Model parameters and results for the compressibility $K$ and $b^{*} / b$. The parameters $g_{\omega}=3 g_{q \sigma}$ and $g_{\sigma}=3 g_{q \sigma}$ are adjusted to $E / A-M_{N} \simeq-15.75 \mathrm{MeV}$ at $k_{F} \simeq 1.36 \mathrm{fm}^{-1}$.

\begin{tabular}{lccr|cc}
\hline \hline Model & $b(\mathrm{fm})$ & \multicolumn{1}{c|}{$g_{\sigma}^{2}$} & \multicolumn{1}{c|}{$g_{\omega}^{2}$} & $K(\mathrm{MeV})$ & $b^{*} / b$ \\
\hline (1) QHD (MFT) & - & 105.96 & 161.10 & 545 & - \\
$(2) \mathrm{H}$ & 0.6 & 117.00 & 90.27 & 330 & 1.045 \\
$(3) \mathrm{H}$ & 0.7 & 112.10 & 98.80 & 340 & 1.05 \\
$(4) \mathrm{H}+\mathrm{EXCH}(\sigma+\omega)$ & 0.6 & 152.89 & 88.43 & 370 & 1.06 \\
$(5) \mathrm{H}+\mathrm{EXCH}(\sigma+\omega+\pi)$ & 0.6 & 165.60 & 90.90 & 362 & 1.07 \\
$(6) \mathrm{H}+\mathrm{EXCH}(\sigma+\omega+\pi+\rho)$ & 0.6 & 165.66 & 89.80 & 351 & 1.07 \\
$(7) \mathrm{H}+\mathrm{EXCH}\left(\sigma+\omega+\pi_{\text {att }}\right)$ & 0.6 & 147.44 & 98.75 & 353 & 1.06 \\
$(8) \mathrm{H}+\mathrm{EXCH}\left(\sigma+\omega+\pi_{\text {att }}+\rho_{\text {att }}\right)$ & 0.6 & 137.71 & 101.50 & 340 & 1.05 \\
\hline \hline
\end{tabular}
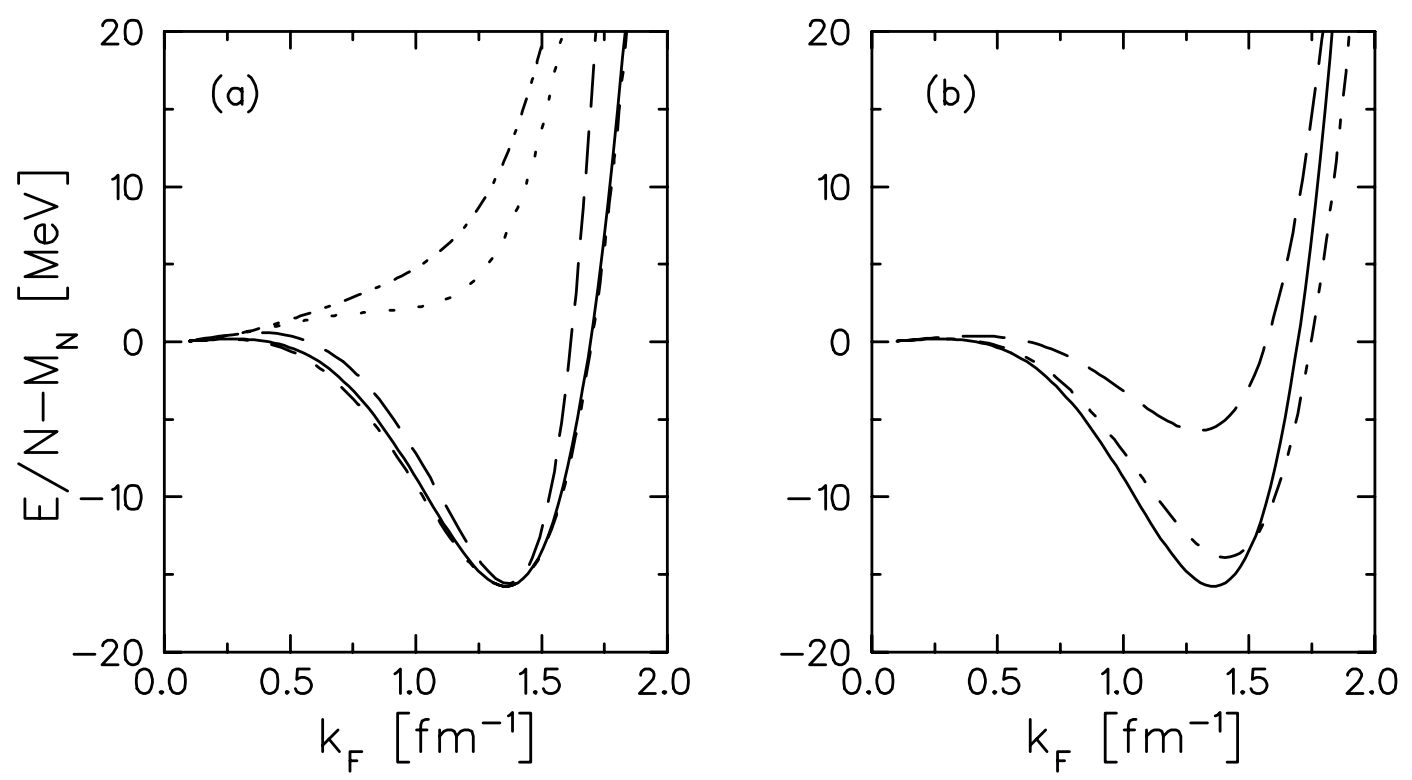

FIGURE 1. The total energy per particle as a function of the Fermi momentum. (a) The long-dashed curve corresponds to QHD (MFT). The long-short-dashed curve is our Hartree result, parameter set (2) in Table I, and the solid curve is for parameter set (6). The shortdashed (dot-dashed) curve is calculated with the $\mathrm{H}$ couplings (2) including exchange terms of $\sigma+\omega(\sigma+\omega+\pi)$. (b) The solid curve is for parameter set (8), with the zero-range parts of $\pi$ and $\rho$ removed. The long-dashed (dash-dotted) is calculated with the $\mathrm{H}$ couplings (2) and exchange terms of $\sigma+\omega+\pi_{\text {att }}\left(\sigma+\omega+\pi_{\text {att }}+\rho_{\text {att }}\right)$. All curves are for $b=0.6 \mathrm{fm}$. 